\title{
Nachweis der Ermüdungssicherheit von Brücken - Teil 1: Veranlassung, Ziel und Messkonzept des Monitoring-Projekts „Bahnbrücke Eglisau“
}

Durch steigende Anforderungen des modernen Bahnverkehrs (vor allem durch höhere Achslasten und Zugfrequenzen) werden Bahnbrücken immer stärker beansprucht. Entsprechend muss die Tragund Ermüdungssicherheit dieser Brücken nachgewiesen werden. Dies erfolgt über eine so genannte Aktualisierung, indem die effektiv auftretende Beanspruchung des Brückentragwerks genau ermittelt wird. Im Rahmen eines Pilotprojekts werden zurzeit zerstörungsfreie, statische und dynamische Belastungsversuche an der Bahnbrücke Eglisau, Schweiz, durchgeführt, um Informationen zu erhalten, auf deren Basis zuverlässige Aussagen zur Tragsicherheit und Ermüdungssicherheit gemacht werden sollen. Im laufenden Bahnbetrieb ist die eingleisig befahrene Bahnbrücke schwer zugänglich, weshalb die Langzeitmessungen mit Hilfe eines festinstallierten, vollautomatischen Bauwerk-Monitoring-Systems (swissMon) durchgeführt werden. Mit Hilfe dieses Systems können beliebig viele, unterschiedliche Messsensoren zyklisch oder getriggert durch das Überfahren eines Zuges betrieben werden. Die mit swissMon gewonnen Daten sind eine verlässliche Grundlage für weitere Analysen und Modellrechnungen und unterstützen die beteiligten Tragwerksingenieure insbesondere bei der Beurteilung der Ermüdungssicherheit.

Fatigue design of bridges - Part 1: Motive, intent and measuring concept of the Swiss monitoring-study "Railway Bridge Eglisau. Due to increasing requirements of modern railways (higher load per axle, higher train frequencies etc.) higher loads are drawn on railway bridges in the present. A pilot study has been carried out to capture non-intrusive static and dynamic load tests on a railway bridge at Eglisau, Switzerland. The tests have provided reliable data needed to produce a meaningful report presenting the current and predicted structural safety, serviceability and structural fatigue of the bridge. The single track railway bridge provides limited access when in operation. Therefore, a fully automated construction monitoring system (swissMon) was installed onto the bridge to continuously supply remote measurement data throughout the monitoring period. The system was used to program multiple sensors and sensor types to record measurements at regular time intervals or to trigger the sensors upon the passing of a train. The data obtained from swissMon provided the fundamental reliable information required to carry out further analysis and modeling to support the expert engineers' structural assessment of the single track railway bridge at Eglisau.

\section{Einleitung}

Durch steigende Anforderungen des modernen Bahnverkehrs (vor allem durch höhere Achslasten und Zugsfrequenzen) werden die Bahnbrücken sukzessive stärker be- ansprucht. Dies stellt die Brückeneigner zunehmend vor die Aufgabe, ihren Bauwerksbestand im Hinblick auf die Sicherheit und die daraus abgeleitete Restnutzungsdauer zu bewerten. Für die weitere Nutzung dieser Brücken sind deshalb zuverlässige Aussagen bezüglich Tragsicherheit, Gebrauchstauglichkeit und Ermüdungssicherheit notwendig. Aktuelle Informationen bezüglich der Bauwerkseigenschaften liegen aber oft nur unzureichend oder unvollständig vor. Anhand des folgenden Beispiels wird aufgezeigt, wie die geforderten Informationen mit einem leicht anpassbaren, modularen Messkonzept genau und zuverlässig gewonnen werden können.

\section{Beschreibung der Brücke}

Die Bahnbrücke Eglisau wurde im Zeitraum von 1895 bis 1897 durch die ehemalige Schweizer Nordostbahn erstellt (Bild 1). Sie verbindet auf der Strecke Bülach-Schaffhausen eingleisig die Stationen Eglisau und Hüntwangen-Wil über den Rhein.

Im zentralen Bereich des 457 m langen Bauwerkes befindet sich eine über $90 \mathrm{~m}$ frei tragende, genietete Stahlfachwerkkonstruktion. Nördlich und südlich der $9 \mathrm{~m}$ hohen Fachwerkkonstruktion schließen sich die beiden aus Kalksteinquadern gemauerten Vorlandbrücken an, deren Stützen im Bereich der Auflagerbereiche bis zu $50 \mathrm{~m}$ hoch sind. Die Gewölbeweiten der Vorlandbrücken liegen zwischen $12 \mathrm{~m}$ und $15 \mathrm{~m}$.

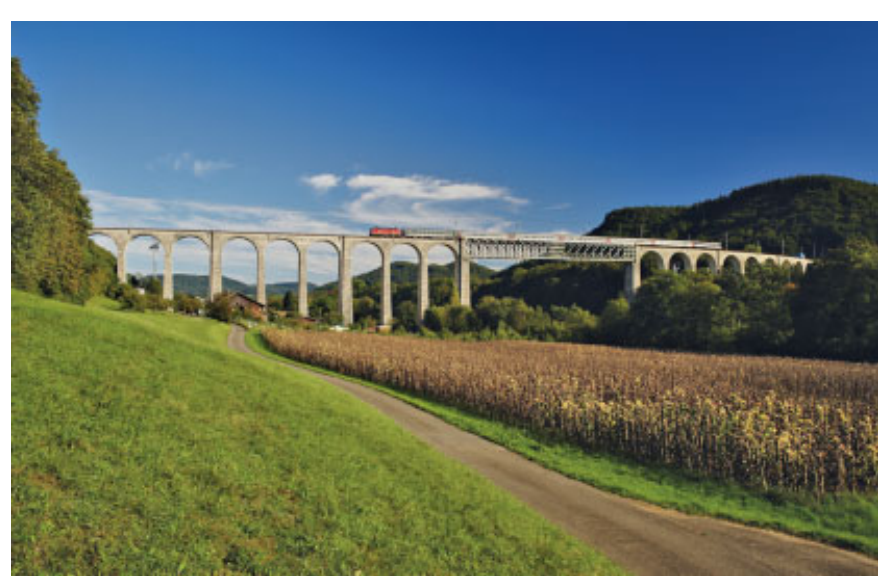

Bild 1. Gesamtansicht der Eisenbahnbrücke Eglisau inklusive der Vorlandbrücken und Stahlfachwerk

Fig. 1. Overall view of the Eglisau Railway Bridge 
Die Bahnbrücke Eglisau blickt auf eine bewegte Vergangenheit zurück. Bereits unmittelbar nach ihrer Fertigstellung wurden an den beiden Gewölben neben der Hauptöffnung Rissbildungen und Senkungen im Scheitel sowie horizontale Bewegungen der Uferpfeiler in Richtung der Fachwerkbrücke festgestellt. Bereits damals wurde daraufhin ein Monitoring-System installiert, um das Tragwerksverhalten genauer zu erfassen. Infolge dieser Deformationen musste das bewegliche Lager mehrfach nachgestellt werden. Im Jahr 1921 wurde auf Seite Hüntwangen, wo sich das bewegliche Lager befindet, eine Verspannvorrichtung eingebaut, die mittels Hebelwirkung der horizontalen Verschiebung der Uferpfeiler entgegenwirkt.

Basierend auf den Ergebnissen einer im Jahr 1980 durchgeführten statischen Überprüfung waren umfangreiche Verstärkungsarbeiten notwendig, die 1982/83 realisiert wurden. Neben Verstärkungsmaßnahmen bei den Stabanschlüssen wurde insbesondere die bestehende Fahrbahn aus Zoreseisen durch einen Schottertrog ersetzt.

\section{Veranlassung}

Eine 2001 bis 2003 im Auftrag der SBB durchgeführte Überprüfung der Stahlkonstruktion (Bild 2) hat zu dem Ergebnis geführt, dass die statische Ausnutzung der Stahlkonstruktion sehr hoch sei. Die Trag- und Ermüdungssicherheit konnte hierbei für einige Bauteile rechnerisch nicht nachgewiesen werden. In Folge dieser Berechnungsergebnisse wären weitere Verstärkungsmaßnahmen erforderlich gewesen, die aufgrund der Lage und Zugänglichkeit am Objekt sowie der betrieblichen Randbedingungen auf der eingleisigen Strecke nur mit großem technischem und finanziellem Aufwand hätten ausgeführt werden können. Diese Maßnahmen stellen für den Bauwerkseigentümer keine bevorzugte Option dar.

Bislang durchgeführte Tragwerksanalysen basierten ausschließlich auf theoretischen Berechnungen und Erfahrungswerten. Davon ausgehend, dass diese Untersuchungen insbesondere bezüglich Ermüdungssicherheit entsprechende Unschärfen und Ungenauigkeiten beziehungsweise nicht quantifizierbare Sicherheitsreserven beinhalten, wurde von der Schweizerischen Bundesbahnen SBB die Entscheidung getroffen, die Annahmen hinsichtlich der Tragwerksbeanspruchungen zu überprüfen. Die

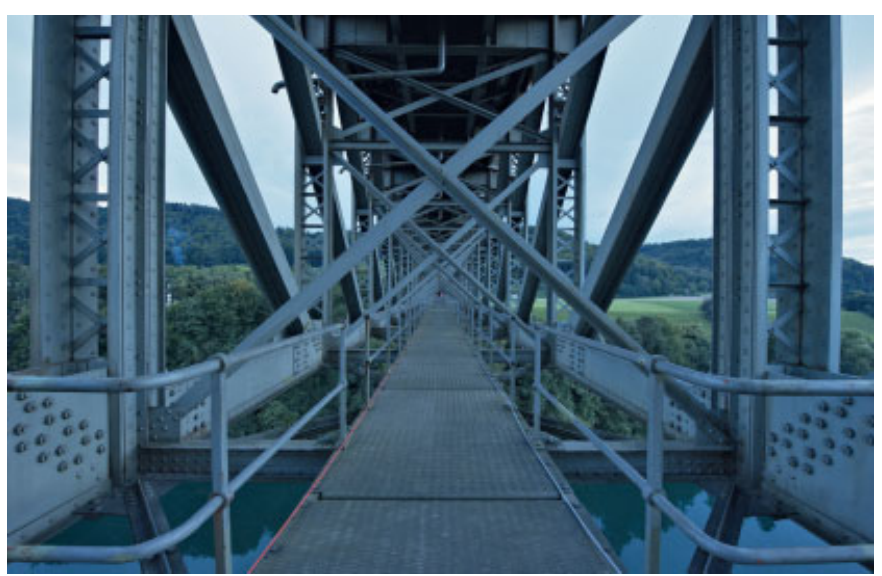

Bild 2. Inspektionsgang auf der unteren Ebene des Stahlfachwerkes

Fig. 2. Lower inspection gallery verwendeten Tragwerksmodelle wurden verfeinert, um die Nachweise der Trag- und Ermüdungssicherheit anhand möglichst wirklichkeitsnaher Informationen über die Beanspruchung des Tragwerks durchzuführen.

Anders als bei den bisherigen Untersuchungen sollte diese Überprüfung durch ein umfassendes Messprogramm abgestützt werden, mit dem die Beanspruchung des Brückentragwerks durch Bahnlasten möglichst präzise erfasst werden sollte.

\section{Projektziele}

Das generelle Ziel des Projektes „Monitoring Bahnbrücke Eglisau“ (Bild 3) ist die Beurteilung des tatsächlichen Tragverhaltens anhand einer messtechnischen Erfassung von Dehnungen und Verschiebungen an charakteristischen Stellen der Konstruktion.

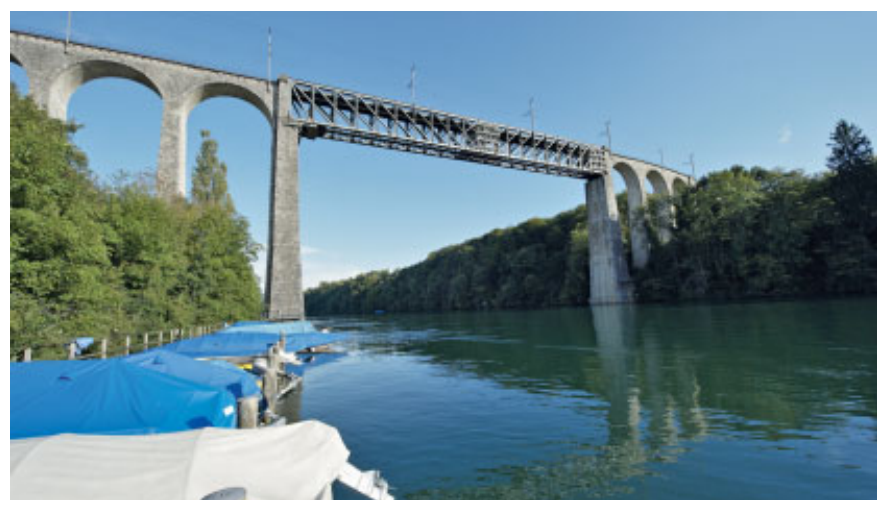

Bild 3. Stahlkonstruktion der Brücke Eglisau mit den im zentralen und rechten Bereich montierten Baugerüsten für die Sensorinstallation

Fig. 3. Steel part of the Eglisau bridge showing installation scaffolds in the central and right part

Mit Hilfe messtechnisch abgesicherter Berechnungsannahmen und eines optimierten Berechnungsmodells sind Nachweise der Tragsicherheit, der Gebrauchstauglichkeit und der Ermüdungssicherheit zu erbringen. Seitens des Auftraggebers besteht die Hoffnung, dass aufgrund dieser Nachweise die Brücke als sicher beurteilt werden kann, so dass auf aufwändige Verstärkungen des Tragwerks verzichten werden kann.

Neben den genannten Zielen wird mit der Umsetzung des Projektes noch ein zusätzliches Fernziel verfolgt. Auf der Grundlage des aktuellen Wissenstands ist davon auszugehen, dass sich die Restlebensdauer bestehender Bauwerke durch die Kombination von theoretischen Betrachtungen und messtechnischen Untersuchungen genauer abschätzen und sich folglich zukünftig der Aufwand für Erhaltungsmaßnahmen reduzieren lässt. Das Projekt wird deshalb auch als Pilotprojekt genutzt, um neue Erkenntnisse für effiziente, messtechnische Überwachungskonzepte von Kunstbauten oder ähnlichen Objekte zu liefern.

\section{Projektphasen}

Vor dem Hintergrund der formulierten Ziele wurde das Anfang 2010 gestartete und auf einen Zeitraum von etwa zwei Jahren ausgelegte Projekt in vier Phasen gegliedert. 


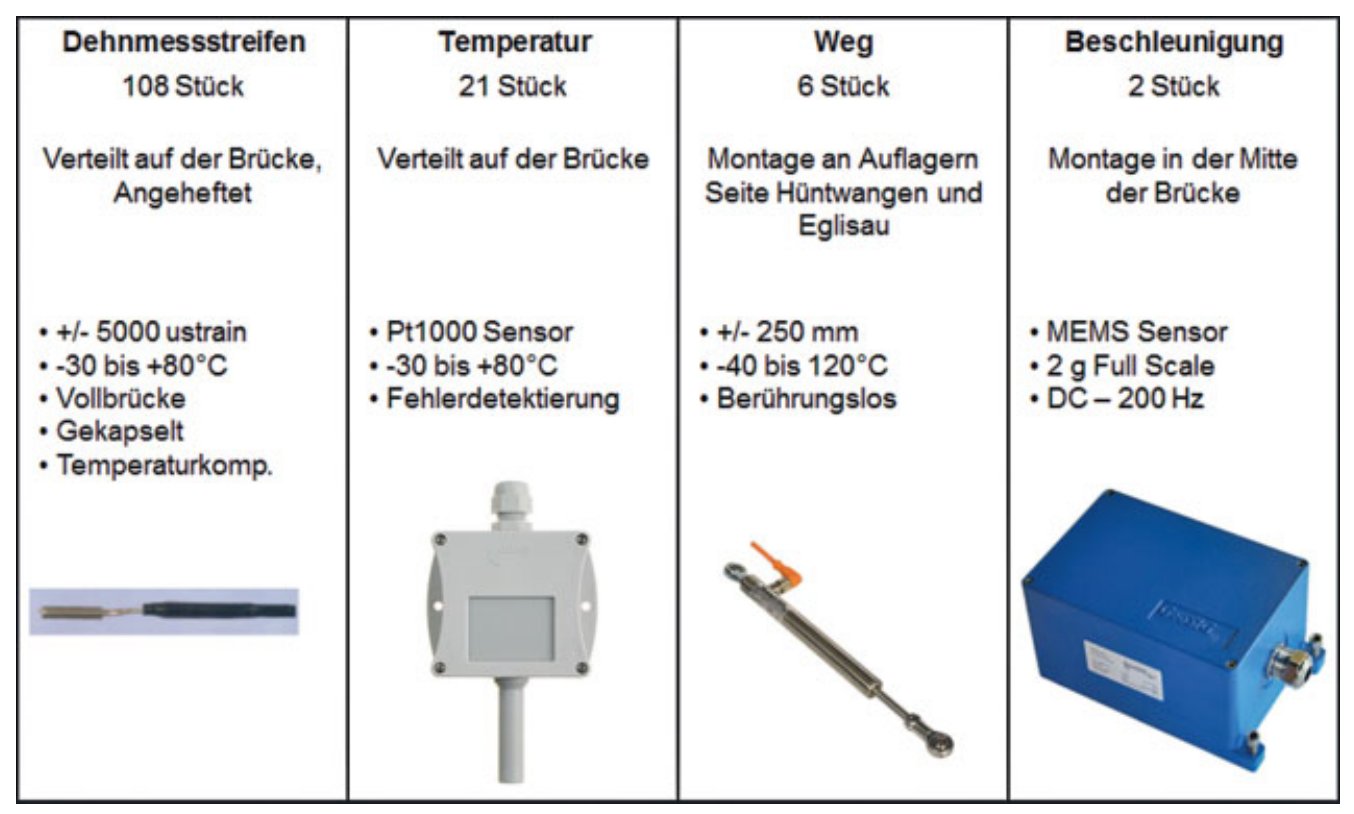

Bild 4. Im Projekt verwendete Sensoren

Fig. 4. Sensors used within the project
Aufbauend auf einer detaillierten Sichtung und Beurteilung der vorhandenen Unterlagen, wurden in der ersten Projektphase die Anforderungen für die Nachweisführung definiert und ein räumliches Berechnungsmodell für die statischen Betrachtungen erarbeitet. Abgestimmt auf dieses Berechnungsmodell wurde das der Ausschreibung zugrunde liegende Messkonzept überprüft und optimiert. Darüber hinaus wurden in dieser Phase die endgültigen Anforderungen an die erforderliche Messtechnik und die bei der Installation zu berücksichtigenden Randbedingungen definiert. Ein besonderes Augenmerk lag hierbei auf der Untersuchung der Auswirkungen des erst 1982 eingebauten Schottertroges auf das Tragverhalten des Bauwerks.

Nach der Prüfung und Freigabe des Ausführungsprojektes durch die SBB wurde in der zweiten Projektphase die definierte Messtechnik geliefert und installiert. Hierbei wurde ein modernes, digitales Datenerfassungssystem in Kombination mit robuster und leistungsstarker Sensorik aufgebaut. Die Installation der insgesamt 137 Sensoren wurde im Sommer 2010 ausgeführt und konnte, trotz der schwierigen, logistischen Randbedingungen, in nur drei Wochen erfolgreich abgeschlossen werden (Bild 4). Ausschlaggebend hierfür war insbesondere die für die Installation der Sensoren gewählte Befestigungstechnik. In Abstimmung mit den am Projekt Beteiligten konnte von einer aufwändigen Klebetechnik abgesehen und stattdessen ein speziell an die Rahmenbedingungen angepasstes Heftverfahren angewendet werden (Bild 5). Neben dem Zeitgewinn und folglich auch Kostenersparnissen ermöglichte diese Entscheidung auch den Einsatz von Sensoren, die werkseitig unter kontrollierten Verhältnissen gekapselt wurden. Dies hat sich sehr positiv auf die Qualität und Nachhaltigkeit der Installation ausgewirkt. Die Ausfallrate der Sensoren ist über den gesamten Messzeitraum als vernachlässigbar gering zu bewerten.

Im Herbst 2010 wurden im Rahmen der dritten Projektphase Belastungsversuche (Bild 6) mit dem Ziel ausgeführt, die Funktionstüchtigkeit des installierten LangzeitMesssystems zu überprüfen und die Messwerte aus definierten Einwirkungen und den theoretisch am Modell ermittelten Rechenwerten gegenüberzustellen. So konn-

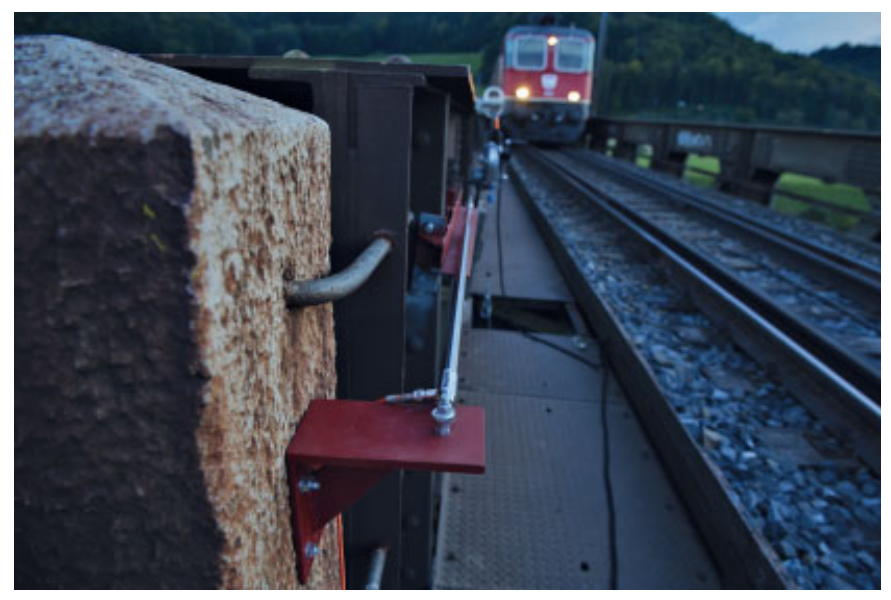

Bild 5. Am Widerlager Hüntwangen installierter Wegmessgeber

Fig. 5. Extensometer installed at the Hüntwangen abutment

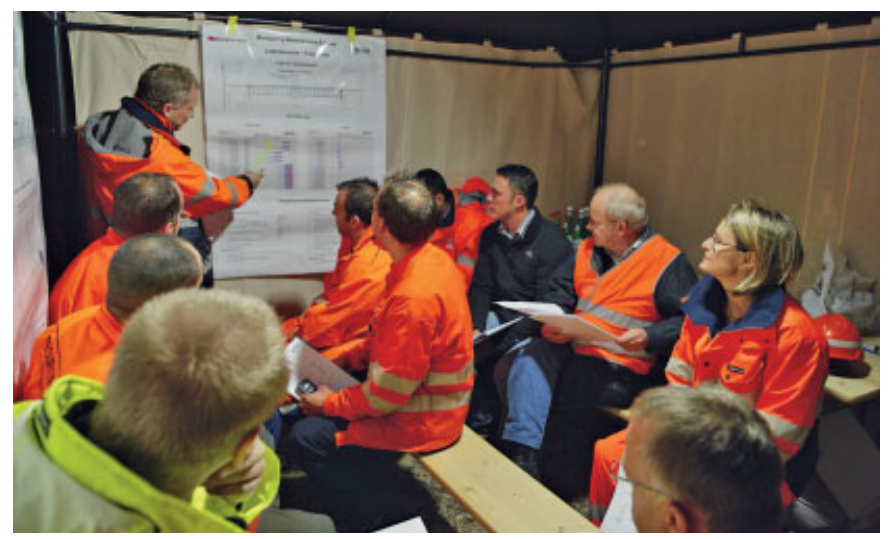

Bild 6. Instruktion aller Projektbeteiligter unmittelbar vor Beginn der Belastungsversuche

Fig. 6. Load Test Briefing

ten Schlussfolgerungen hinsichtlich der Effizienz des gewählten Messkonzeptes abgeleitet werden. Die Belastungsversuche wurden auf das im Rahmen des Ausführungsprojektes entwickelte Tragwerkmodell abgestimmt. Es wurden sowohl statische als auch dynamische Belas- 


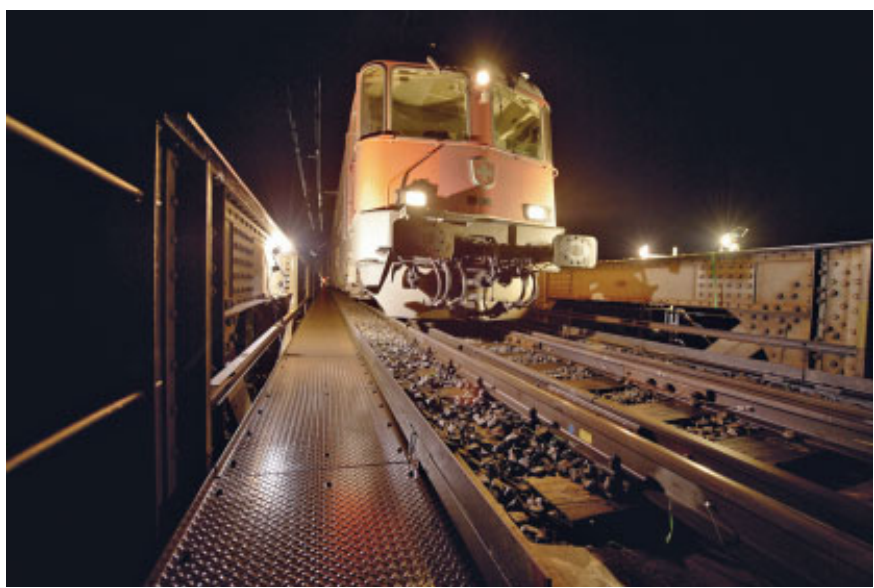

Bild 7. Lokomotivzug auf Lastposition 10 während der Belastungsversuche

Fig. 7. Haul Engine at load position No 10 during load test

tungsversuche ausgeführt. Für die Belastungsversuche kamen drei Lokomotiven mit einer nominellen Last von je $1200 \mathrm{kN}(6$ Achsen à $200 \mathrm{kN})$ zum Einsatz. Für die statischen Belastungsversuche wurden die als Lasten eingesetzten Lokomotiven an definierten Stellen auf der Brücke positioniert (Bild 7) und die hierbei auftretenden Tragwerksverformungen für sieben Lastfälle gemessen. Bei den dynamischen Belastungsversuchen fuhr eine Lokomotive mit konstanter Geschwindigkeit über die Brücke. Im Ergebnis der aufschlussreichen Belastungsversuche wurde das Messkonzept im Hinblick auf die nachfolgende Langzeitmessung angepasst und optimiert.
Die vierte Projektphase beinhaltet die kontinuierliche Erfassung von Messdaten über einen Zeitraum von einem Jahr und wird planmäßig im Frühjahr 2012 abgeschlossen. Parallel hierzu wird an den Nachweisführungen gearbeitet und die Schlussdokumentation inklusive Empfehlungen zum weiteren Vorgehen erstellt.

\section{Projektteam}

Die im Vorfeld durchgeführte Analyse hat schnell zum Ergebnis geführt, dass dieses Projekt nur mit einem spezialisierten, interdisziplinären Projektteam realisiert werden kann. Im Zentrum des Projektteams stehen der für das Tragwerk und die Nachweisführungen zuständige Bauingenieur sowie das für die messtechnische Umsetzung und Messdatenauswertung verantwortliche Monitoring-Unternehmen. Unter Berücksichtigung des neuartigen Charakters und des hohen fachlichen Anspruches des Projekts wurden zusätzlich zwei Berater für die wissenschaftliche und fachtechnische Begleitung hinzugezogen. Die im Einzelnen am Projekt hauptsächlich beteiligten Personen und deren Funktion sind im folgenden Organigramm (Bild 8) dargestellt.

\section{Messkonzept}

Das Messkonzept ist darauf ausgerichtet, die Trag- und Ermüdungssicherheit der Brücke unter Verkehr nachzuweisen. $\mathrm{Zu}$ diesem Zweck werden Stahldehnungen an Tragwerksteilen in Feldmitte, im Auflagerbereich sowie an der Verspannvorrichtung erfasst. Insgesamt wurden dazu 129 voll gekapselte Dehnungsmessgeber in Vollbrückenschal-

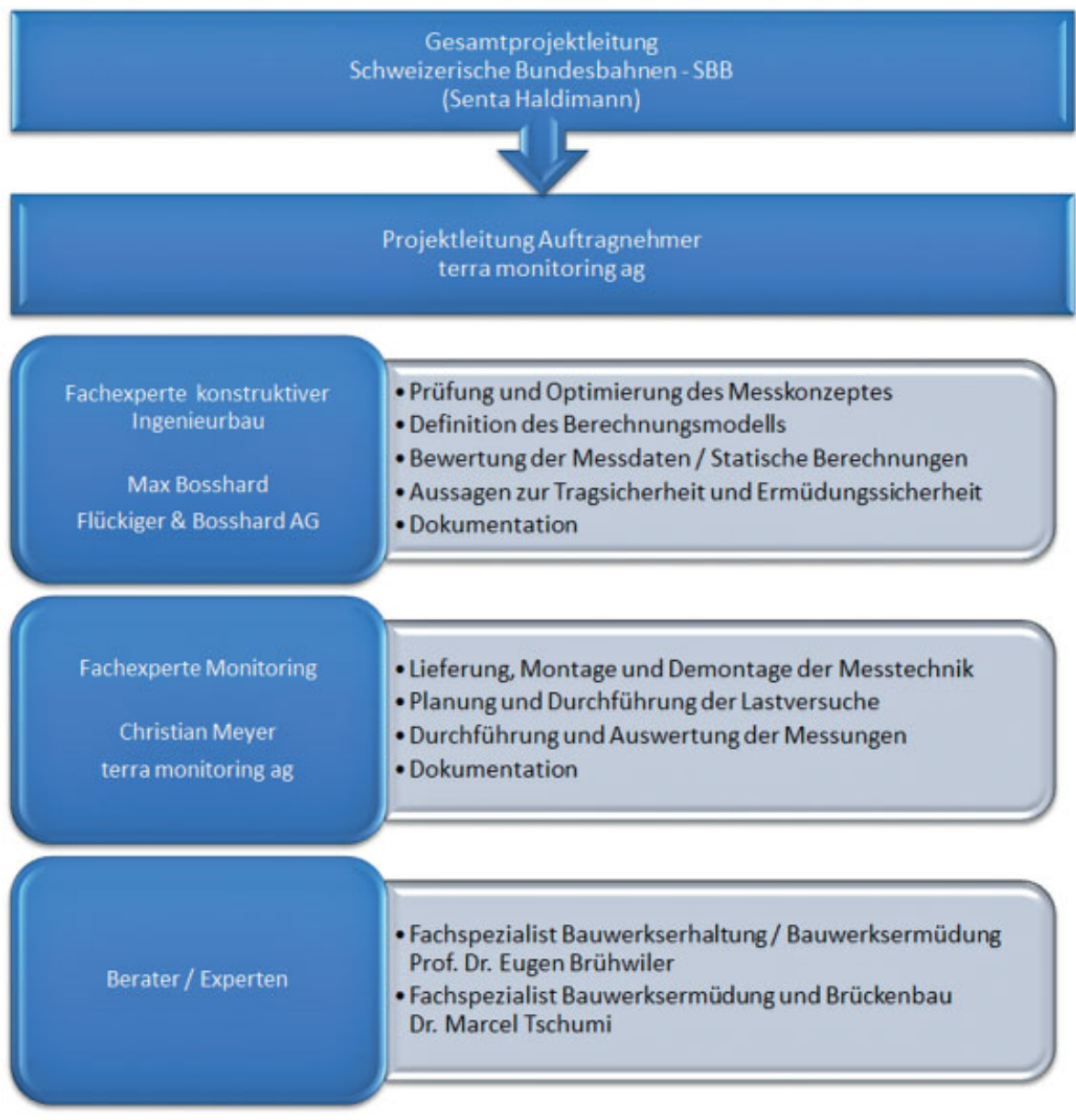

Bild 8. Projektorganisation

Fig. 8. Project organisation 
A
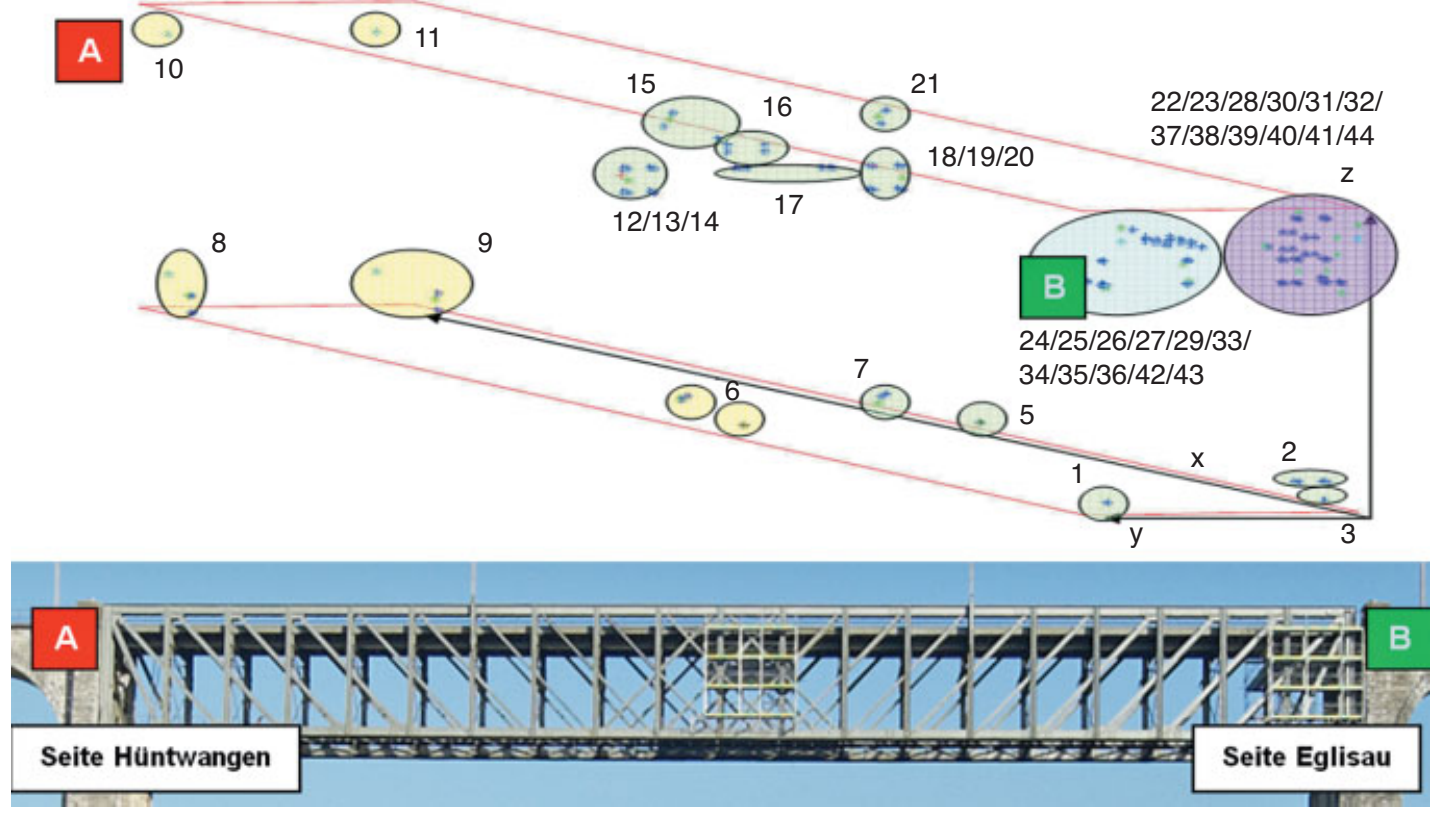

Bild 9. Schematische Übersicht der Lage und Gruppierung der Sensoren

Fig. 9. Scheme showing positioning of the sensors

tung installiert. Um Bewegungen des Stahlfachwerkes bezogen auf die Widerlager erfassen zu können, wurden sechs Wegmessgeber im Bereich des Ober- bzw. Untergurtes installiert (Bild 9). Die Messanordnung wird durch Beschleunigungsmessgeber und Temperatursensoren vervollständigt.

Die für die Umsetzung des Messkonzeptes erforderliche Messtechnik muss einem hohen Qualitätsstandard sowohl hinsichtlich der eingesetzten Sensoren als auch bezüglich der Datenerfassung, Übertragung und Auswertung genügen. Unter Berücksichtigung des Einsatzes auf einer den Witterungseinflüssen ungeschützt ausgesetzten Brücke mit laufendem Bahnverkehr und der geplanten Messdauer von einem Jahr musste darüber hinaus bei der Auswahl der Messsensoren auf eine entsprechende Robustheit sowie hohe System- und Datenverfügbarkeit geachtet werden.

Für eine möglichst störungsfreie und genaue Messdatenerfassung wurde ein digitales Datenbussystem eingesetzt. Die A/D-Wandlung der Messsignale erfolgt hierbei nah am Sensor, um störungsanfällige analoge Datenleitungen so kurz wie möglich und den fehleranfälligen Verkabelungsaufwand so gering wie möglich zu halten.

Die Messungen werden entsprechend dem, von der terra monitoring ag entwickelten und für das Projekt erweiterte, swissMon-Messkonzept ausgeführt (Bild 10). Die auf der vor Ort stationierten Datenerfassungseinheit laufende Software überwacht kontinuierlich alle angeschlossenen Sensoren. Sobald ein Zug auf die Brücke fährt, wird dies durch swissMon automatisch erkannt (Bild 11). Die Überfahrt wird unter Berücksichtigung eines definierten Pre- und Post-Event-Zeitfensters vollständig aufgezeichnet. Sobald der Zug die Brücke wieder verlassen hat, werden die Daten abgespeichert und automatisch ausgewertet. Die Auswertung umfasst die Berechnung von statistischen $\mathrm{Pa}$ rametern (Standardabweichung, Minimal- und Maximalwerte, RMS, Mittelwert) und eine Rainflow-Analyse. Mittelwerte und Standardabweichungen werden nach einer Basislinienkorrektur durchgeführt. Die Basislinie wird aus

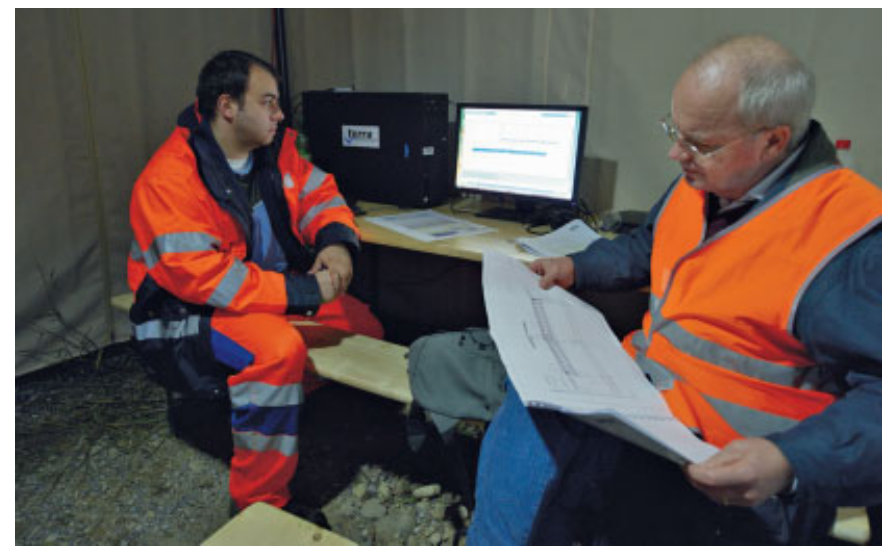

Bild 10. Online Datenauswertung und Bewertung der Ergebnisse während der Belastungsversuche

Fig. 10. Online data reduction an analysis during load tests

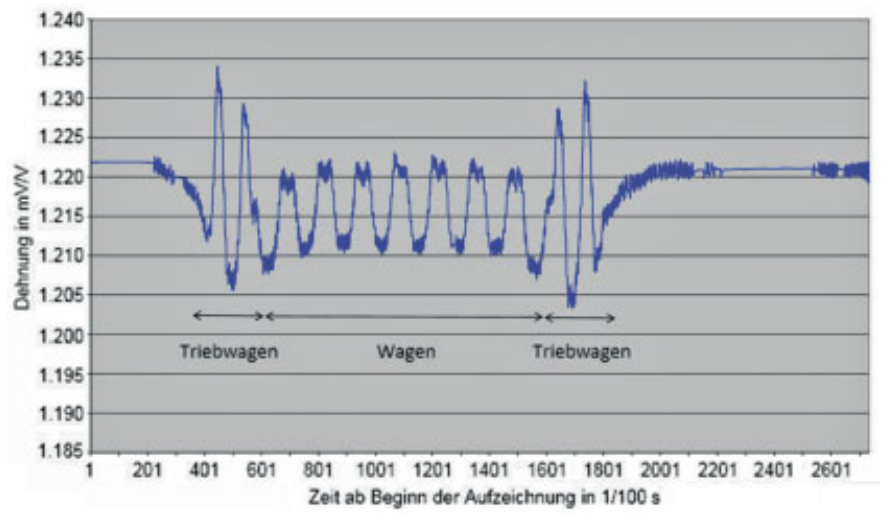

Bild 11. Messsignal eines Dehnungsmessgebers während einer S-Bahn-Überfahrt

Fig. 11. Measuring signal of a strain gauge while commuter train is crossing the bridge

dem Mittel der ersten 1000 Datensätze errechnet, danach werden die Absolutwerte auf die Basislinie als neuen Nullwert reduziert. Die Werte für Minimum, Maximum und RMS werden vor der Basislinienkorrektur berechnet. 


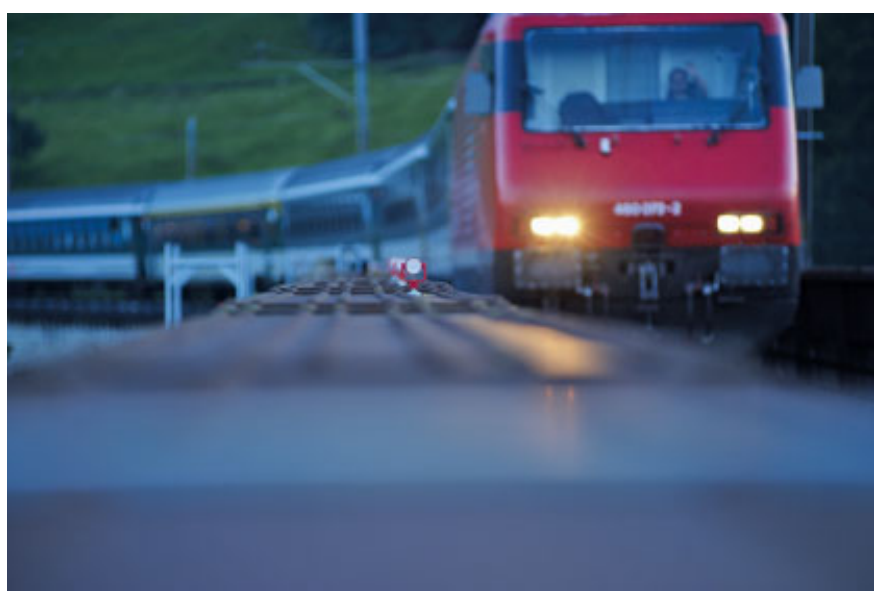

Bild 12. Freie Fahrt auf der Rheinbrücke Eglisau dank messtechnischer Überwachung

Fig. 12. Clear line for trains crossing the Eglisau bridge granted by monitoring

Die ausgewerteten Messdatensätze werden auf den Projektserver übertragen, dort redundant gesichert und für die Visualisierung sowie für die weitere Bearbeitung bereitgestellt. Mit dem umgesetzten Messkonzept konnten sowohl das generelle Verhalten der Bahnbrücke Eglisau als auch das nur lokal auftretende Verhalten in Feldmitte bzw. im Auflagerbereich erfasst und beurteilt werden.

\section{Ausblick}

Die Langzeitmessungen des Projektes „Monitoring Eisenbahnbrücke Eglisau“ dauern weiterhin an (Bilder 12 und 13), so dass eine abschließende Bewertung noch aussteht. Im zweiten Teil dieses Projektberichts, der zu einem späteren Zeitpunkt veröffentlicht wird, werden die dann vorliegenden Daten detailliert analysiert und kommentiert sowie die Ergebnisse des Ermüdungsnachweises erläutert.

Nicht zuletzt auch aufgrund der Auswertung der oben beschriebenen Belastungsversuche kann jedoch bereits jetzt gefolgert werden, dass das umgesetzte Messkonzept die Dehnungen und Verschiebungen an den konkreten Messstellen zuverlässig erfasst und mit der Dauermessung als

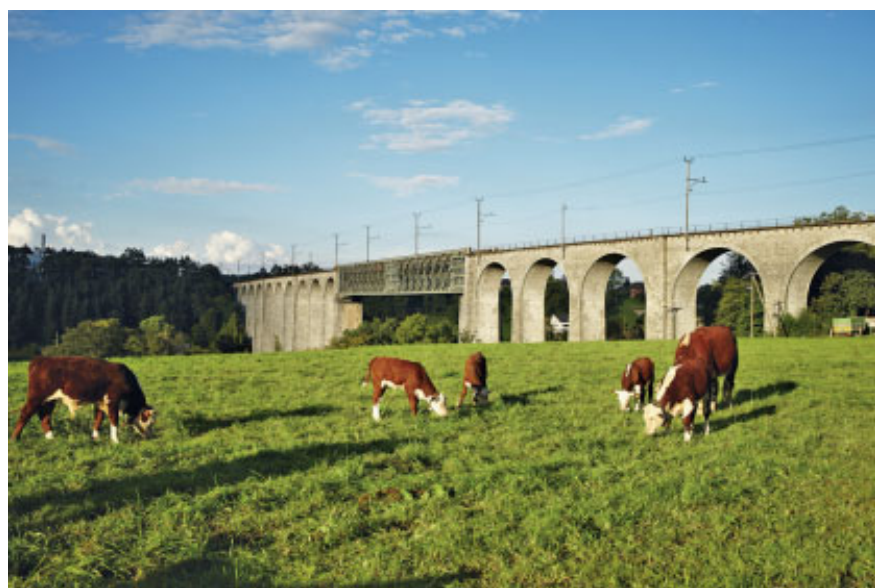

Bild 13. Blick von Eglisau Richtung Hüntwangen

Fig. 13. Eglisau Bridge

Grundlage für die Beurteilung der Ermüdungssicherheit verwendet werden kann.

Grundlage für die erfolgreiche Umsetzung dieses Monitoring-Projektes war die sehr gute Zusammenarbeit aller beteiligten Fachspezialisten. In diesem Zusammenhang ist besonders auf die in allen Projektphasen intensive, offene und fachlich sehr konstruktive Zusammenarbeit mit dem Auftraggeber hinzuweisen, die massgeblich zum guten Gelingen dieses Projekts beigetragen hat.

\section{Bildnachweis:}

alle Bilder privat

\section{Autoren dieses Beitrages:}

Christian Meyer, MSc. Ingenieurgeologie, meyer@terra.ch, terra monitoring ag, Obstgartenstrasse 7, CH-8006 Zürich

Max Bosshard, Dipl.-Bauing. ETH, max.bosshard@fbag.ch, Flückiger \& Bosshard AG, Räffelstrasse 32, Postfach 5070, CH-8045 Zürich

Prof. Dr. Eugen Brühwiler, Dr. és sc. Techn / Dipl. Civil Engineer ETH, eugen.bruehwiler@epfl.ch,

Ecole Polytechnique Fédérale de Lausanne (EPFL),

MCS-ENAC-EPFL, Station 18, CH-1015 Lausanne 